\title{
Development of a variable curvature mirror for the delay lines of the VLT interferometer
}

\author{
M. Ferrari ${ }^{\star}$ \\ Laboratoire d'Optique, Observatoire de Marseille, IGRAP, 2 Pl. Le Verrier, F-13248 Marseille Cedex 4, France
}

Received January 16; accepted March 21, 1997

\begin{abstract}
This paper presents the variable curvature mirror ( $\mathrm{VCM}$ ) serving for beam management purposes in the interferometric mode of the Very Large Telescope (VLT). This special device located in the delay line system provides for a precise positioning of the pupil image of an individual telescope in the recombination laboratory. The particularity of this active mirror is its continuous variable curvature from $(2800 \mathrm{~mm})^{-1}$ to $(84 \mathrm{~mm})^{-1}$ which has to be achieved with a reasonable optical quality. This large curvature variation needed in the system, lead us to extend the classical theory of elasticity to the range of large deformations (i.e. the achieved flexions are larger than the mirror's thickness). This new approach, in the domain of active optics, gave us a theoretical support to determine the physical parameters of the mirror (thickness distribution, loading configuration). Today the VCM system has been thoroughly tested and its performances evaluated. The experimental results have shown good agreements with the theory.
\end{abstract}

Key words: techniques: interferometric instrumentation: miscellaneous - telescopes

\section{Introduction}

In the early 90's the European Southern Observatory issued a call for proposal for a variable curvature mirror system serving for beam management purposes in the interferometric mode of the Very Large Telescope. While the VLTI is tracking an astronomical object, the delaylines provide for the equalization of the optical paths of individual telescopes by varying positions in the interferometic tunnel. The optical system of a delay-line, represented at Fig. 1, is a cat's eye mounted on a carriage and

Send offprint requests to: M. Ferrari

* at present with ESO, Karl-Schwarzschild Strasse 2, D-85748 Garching b. Munchen, Germany. whose location in the tunnel is carefully monitored in order to compensate optical path differences (OPD) up to $120 \mathrm{~m}$ between different telescopes. A parabolic primary mirror produces an image of the source on the secondary and after another reflexion on the primary, the beams are directed to the beam combiner telescope through the interferometric tunnel.

The variable curvature mirror, which is the secondary, is an important component for the achievement of the coherent combined focus. In the VLTI a large field of view (FOV) will be made available and this mode of operation requires a high quality imaging of the pupils. As demonstrated hereafter, the VCM system is critical for the wide FOV operation of the interferometer.

We consider an interferometer with a cat's eye system using a small plane mirror located at its focus as a secondary.

For paraxial rays, a $\delta_{0} / 2$ displacement of the delay-line in the tunnel compensates for a $\delta_{0}$ optical path difference, but for off-axis rays (or "field rays") coming with an angle $i$ this OPD is equal to $\delta_{i}=\delta_{0}\left(1-i^{2} / 2\right)$ (see Fig. 2). Then with a small plane mirror, when the OPD is compensated for paraxial rays, it is not for the rest of the field. But a cat's eye system has the great advantage that rays coming from different parts of the field are physically separated on the secondary mirror. Hence, bending the small mirror with $\delta_{0} . i^{2} / 4$ at the distance $R . i / 2$ from the axis compensates for the "field OPD" $\delta_{0} \cdot i^{2} / 2$ and eliminates the difference between paraxial and off-axis rays (see Fig. 2). This bending corresponds physically to make spherical the small secondary mirror, with a radius of curvature $\rho=R^{2} / 2 \delta_{0}$ where $R$ is the radius of curvature of the cat's eye primary. Then the OPD is compensated for all the FOV and the "field fringes" are eliminated at the combined interferometric focus of the telescopes array. As the radius of curvature $\rho$ of the cat's eye secondary depends of the compensated axial OPD $\delta_{0}$, it is varying during the movement of the delay line system. This possible compensation for the OPD of "field rays" in a cat's eye interferometer has been first noticed by P. Connes (Connes 1956; Connes et al. 1975). 


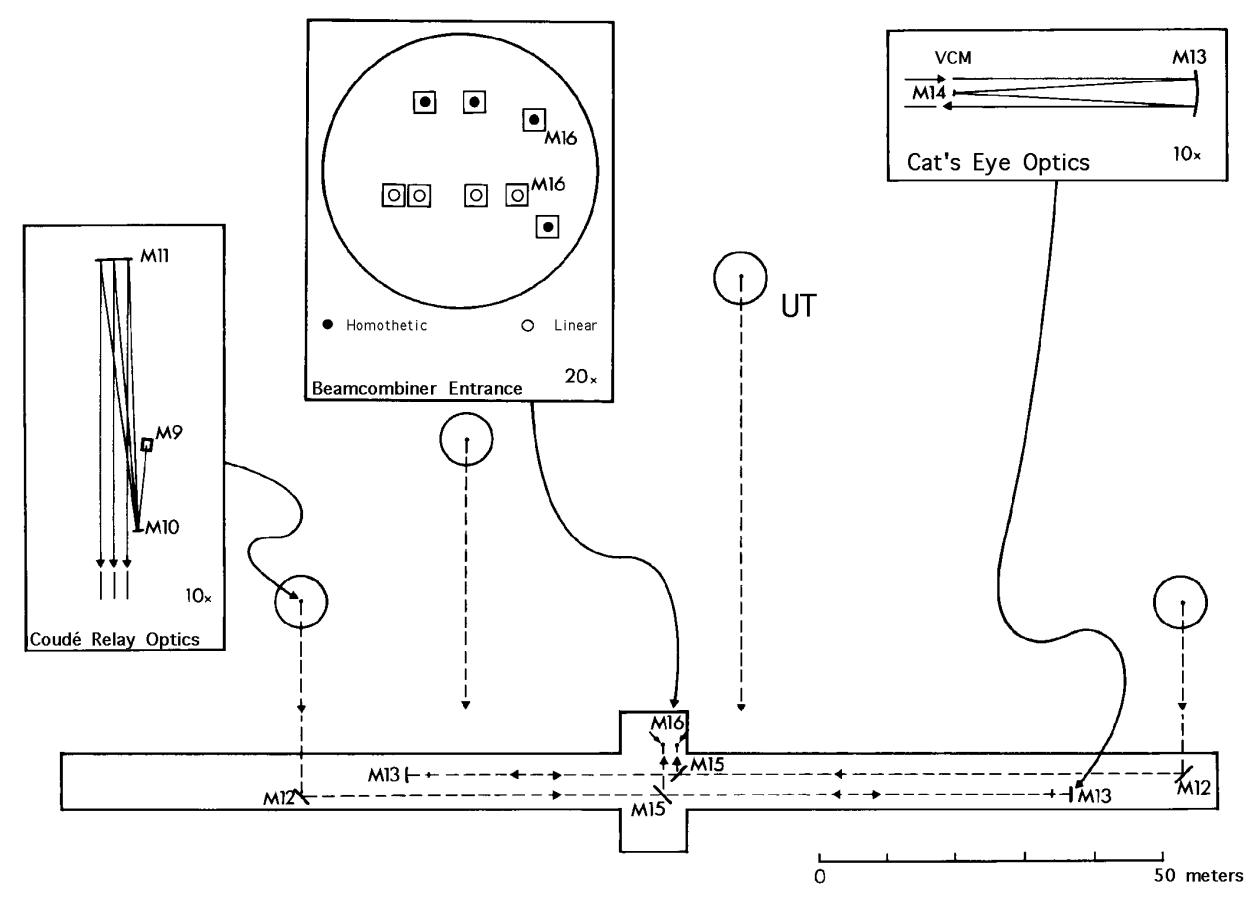

Fig. 1. Optical layout of the VLT Interferometric mode and location of the VCM (M14) in the delay line system

The spherical deformation corresponds also to the one which maintains the center of symmetry $\mathrm{O}$ of the cat's eye system at the same location during the movement (see Fig. 2), the variable focal length then permits to keep a pupil image at a precise location while the delay-line carriage is moving. In order to exactly remap homothetically the output pupil configuration of the telescopes array at the image beamcombiner, an accurate control of the variable curvature, with an error lower than $510^{-3} \mathrm{~m}^{-1}$, is necessary.

Considering the FOV planned for the VLTI (3.5 or 8 arcsec) and the OPD to compensate for, the cat's eye secondary curvature must be continuous variable within a range from $(84 \mathrm{~mm})^{-1}$ to $(2800 \mathrm{~mm})^{-1}$. This has been thoroughly analysed and described by $\mathrm{O}$. von der Lühe (1992a, b) and other authors (Beckers 1991; Jörck et al. 1992), including further phase recoveries with the additional VLTI sub-array, $8 \mathrm{~m}$ Unit Telescopes and Auxiliary Telescopes.

The first approach to variable curvature mirrors belongs to G. Lemaitre and the idea he used was to actively deforme a small metallic mirror with an uniform loading or a central force (Lemaître 1976). A prototype was developed for the European Synchrotron Radiation Facility and served to increase the field of view of a Fourier transform interferometer. The mirror was $20 \mathrm{~mm}$ diameter large and the maximal flexure achieved was $31 \mu \mathrm{m}$, corresponding to a focal ratio varying from $f / 35$ to $f / 20$. The major difference, between this first successful attempt and the ESO prototype, is the amplitude of the maximal deformation applied to the mirror. Due to the range of curvature needed in the delay-line, the simple theory of elasticity was not convenient. We had to extend the theory of elasticity to the large deformations where the total amplitude of the flexure can be of the same magnitude or greater than that of the plate thickness.

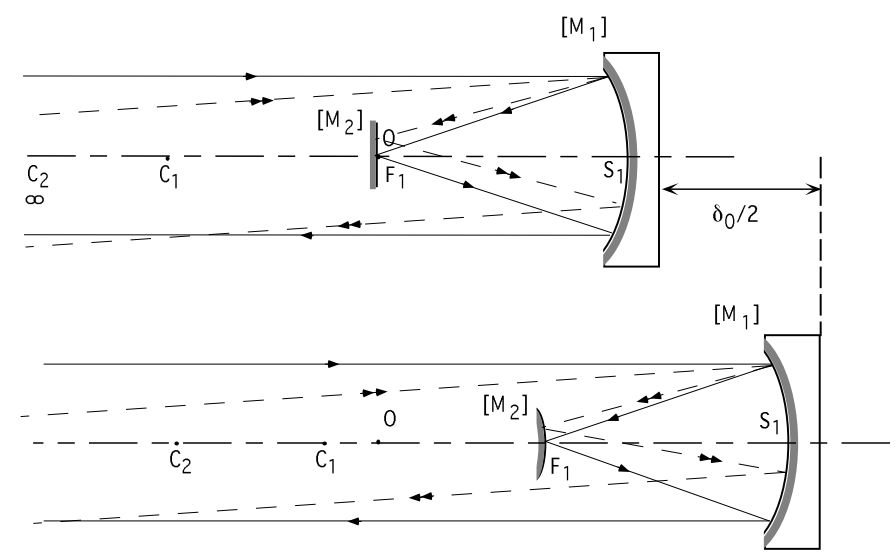

Fig. 2. Field compensation with a Cat's eye system 


\section{Active optics and large deformations}

To generate a peculiar flexure the elasticity problem is to determine the variable thickness distribution associated with a given loading configuration or the reverse, i.e. the loading configuration associated with a constant thickness, solving the elastical equilibrium equations, with the associated boundary conditions (simply supported or clamped edges). In the case of small deformations applied to a circular plate, this process is generally reduced to solve a differential equation between the flexure, the thickness distribution and the loading configuration, one of these last two functions being unknown. But when the flexure become larger, the forces and moments applied to an element generate radial and tangential stress $\sigma_{(r, t)}$ and strains $\varepsilon_{(r, t)}$ in the mirror structure (see Fig. 3). Taking into account these forces we have to solve a differential system with three $3^{\text {rd }}$ order, non-linear, coupled and non-homogeneous equations.

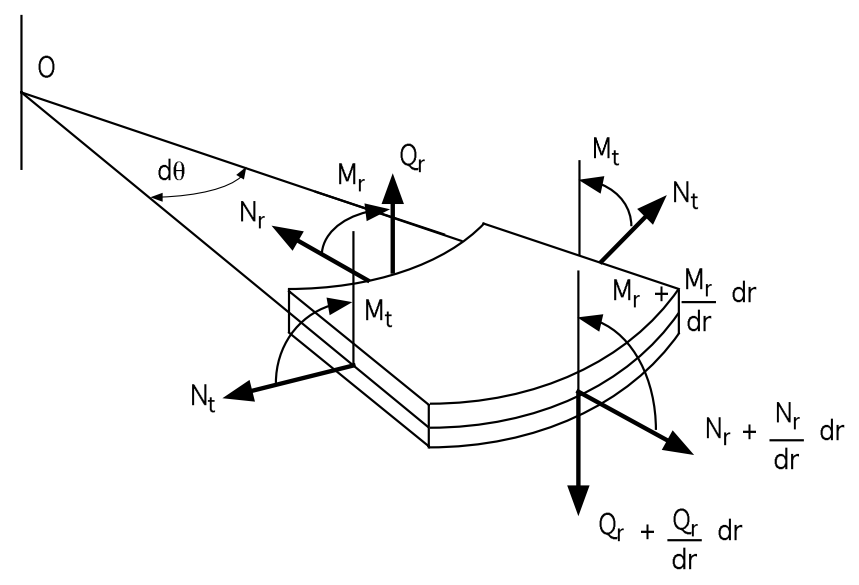

Fig. 3. Forces and moments for an element of the deformed mirror

We consider the quadratic deformation $W(r)=$ $W_{0}\left(1-r^{2} / a^{2}\right)$, where $W_{0}$ is the maximal deformation needed for the variable curvature mirror and $a$ the radius at the edge of the mirror. Using a circular plate with a variable thickness distribution $t(r)$ under an uniform load $q$, the differential system of equilibrium equations can be written as follow:

$$
\begin{aligned}
& M_{r}-M_{t}+r \frac{\mathrm{d} M_{r}}{\mathrm{~d} r}=-\left(N_{r} \frac{\mathrm{d} W}{\mathrm{~d} r}+\frac{q r}{2}\right) r \\
& N_{r}-N_{t}+r \frac{\mathrm{d} N_{r}}{\mathrm{~d} r}=0 \\
& \varepsilon_{t}-\varepsilon_{r}+r \frac{\mathrm{d} \varepsilon_{t}}{\mathrm{~d} r}=-\frac{1}{2}\left(\frac{\mathrm{d} W}{\mathrm{~d} r}\right)^{2}
\end{aligned}
$$

where $M_{(r, t)}$ are the bending moments and $N_{(r, t)}$ the tensiles forces associated (Ferrari 1993).
Using the expression of the radial and tangential stress $\sigma_{(r, t)}$ and strains $\varepsilon_{(r, t)}$ given by Timoshenko \& Woinowsky-Krieger and those of $M_{(r, t)}$ and $\varepsilon_{(r, t)}$, functions of the flexion $W(r)$ and the thickness $t(r)$, this system can be transformed into another one in which the relation between $W(r)$ and $t(r)$ is underlined.

$$
\begin{array}{r}
N_{r}-N_{t}+r \frac{\mathrm{d} N_{r}}{\mathrm{~d} r}= \\
\frac{d^{3} W}{\mathrm{~d} r^{3}}+\left(\frac{1}{r}+\frac{3}{t} \frac{\mathrm{d} t}{\mathrm{~d} r}\right) \frac{\mathrm{d}^{2} W}{\mathrm{~d} r^{2}}+\left(\frac{\nu}{r} \frac{3}{t} \frac{\mathrm{d} t}{\mathrm{~d} r}-\frac{1}{r^{2}}\right) \frac{\mathrm{d} W}{\mathrm{~d} r}= \\
-\frac{12\left(1-\nu^{2}\right)}{E t^{3}} Q_{r} \\
\frac{d^{2} N_{r}}{\mathrm{~d} r^{2}}+\left(\frac{3}{r}-\frac{1}{t} \frac{\mathrm{d} t}{\mathrm{~d} r}\right) \frac{\mathrm{d} N_{r}}{\mathrm{~d} r}-(1-\nu) \frac{1}{t} \frac{\mathrm{d} t}{\mathrm{~d} r} \frac{1}{r} N_{r}= \\
-\frac{E t}{2}\left(\frac{\mathrm{d} W}{\mathrm{~d} r}\right)^{2} \frac{1}{r^{2}}
\end{array}
$$

where the radial shearing force, for an uniform loading configuration, is equal to $Q_{r}=-N_{r} \frac{\mathrm{d} W}{\mathrm{~d} r}-\frac{q r}{2}$.

Behind this point we used a numerical method, integrating the equations, in order to determine the solutions of the differential system. But unlike usual elasticity codes which calculate the deformations of a given structure, we answer the problem backwards. We determine a structural parameter of the mirror, its thickness distribution $t(r)$, from the desired flexure $W(r)$. This new method is well adapted to the problem and permits to obtain solutions fast, avoiding numerous iterations between the inputed structure and the resulting deformation (Ferrari 1993).

The numerical code gives us the thickness distribution, the bending moments, the tensile forces and the total stress to insure that we stay in the elasticity domain of the material. From these results we first can fix the limit of validity of the small deformations approximation. Up to a flexion amplitude equal to $1 / 3$ of the mirror's mean thickness (i.e. a flexion ratio $W_{0} / t_{\mathrm{c}}=1 / 3$ ) both theories give the same thickness distributions with only 2 or $3 \%$ deviations. After this limit, the non-linear effects introduced by the shearing forces into the mirror become important and the differences between the solutions can reach $50 \%$ for a flexion ratio equal to 1.5 (LOOM 1991).

The code calculates also the value of the load $q$ to apply in order to achieve the desired radius of curvature (or the flexion ratio $W_{0} / t_{\mathrm{c}}$ ). This relation is displayed in Fig. 4 in a dimensionless form for stainless steel. This material having a Poisson ratio $\nu$ equal to 0.315 , against 0.20 for a glass, the bending moments and thus the flexion achieved are larger, but the stress are also higher. The limit of the small deflections approximation is evident in this figure where the curves obtained with the two different theories diverge for flexion ratios above $1 / 3$. 


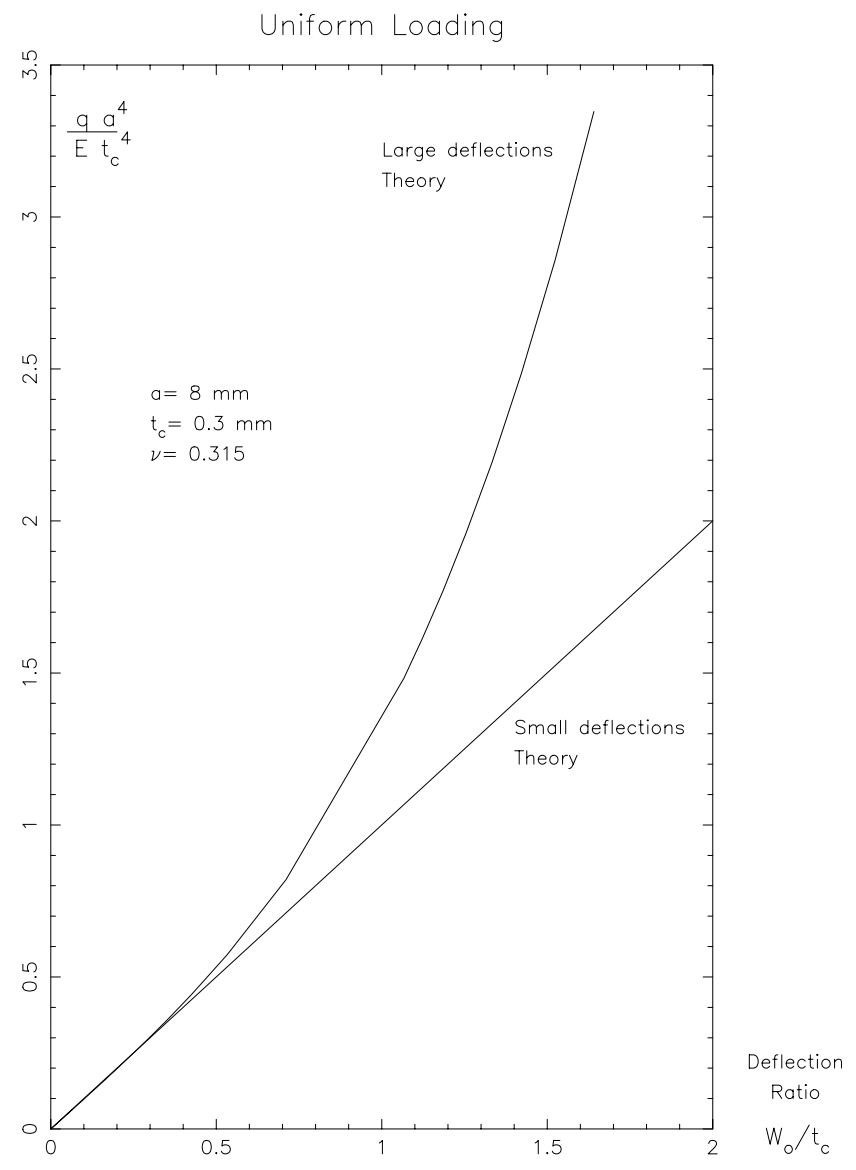

Fig. 4. Load/Flexion relations in the case of a simply supported plate given by the small and large deformations theories

\section{Realization of the prototype}

As seen in Fig. 2, in the cat's eye system the VCM is located between the input and ouput beams of the delay-line and its diameter is then limited by these two beams. The overall diameter of the system cannot exceed $30 \mathrm{~mm}$, and this fact led us to realize a small mirror with a $16 \mathrm{~mm}$ diameter. Starting the curvature variation with a plane mirror to achieve a maximal curvature of $(84 \mathrm{~mm})^{-1}$, this range corresponds to an $f$ ratio varying from $f / \infty$ to $f / 2.6$ and the maximal central flexion achieved is then $380 \mu \mathrm{m}$. Due to the large bending of the mirror, the full domain of curvature is not achievable with a classical optical material as vitroceramic glass (Zerodur). Metal alloys having 100 times higher flexibility than glasses, a stainless steel substrate (AISI 420) has been chosen for this reason and for the fact that deviations to Hooke's law (stress-strain relation) are small with such materials.

The required pressure for the maximal deformation has to be easily achievable with industrial components and this technical constraint led us to choose a very thin meniscus for the active part of the mirror. As displayed in Fig. 4, with a fixed material (i.e. $E$ and $\nu$ fixed), in order to reduce the load necessary to achieve a given deflection $W_{0}$ we have to reduce the thickness $t_{\mathrm{c}}$. But the flexion ratio $W_{0} / t_{\mathrm{c}}$ increases so that it is not possible to obtain a quasilinear relation all along the variation range. A compromise between a small load $q$ and a linear load/curvature relation lead us to chose a meniscus with a $300 \mu \mathrm{m}$ central thickness. With these conditions $\left(a=8 \mathrm{~mm}\right.$ and $\left.t_{\mathrm{c}}=300 \mu \mathrm{m}\right)$ the maximal required load $q$ is lower than $10 \mathrm{daN} / \mathrm{cm}^{2}$ when $R_{\mathrm{c}}=84 \mathrm{~mm}$ and the maximal stress $\sigma_{\max }$ reach 55 $\mathrm{daN} / \mathrm{mm}^{2}$ well below the elastical limit strength of the material $\sigma_{\text {elas }}=120 \mathrm{daN} / \mathrm{mm}^{2}$.

Remembering the amplitude of the deformation achieved at the maximal curvature $\left(W_{0}=380 \mu \mathrm{m}\right)$, the reason why we developed the large deformations theory of elasticity is obvious: The flexion ratio $W_{0} / t_{\mathrm{c}}$ is larger than 1.25 , very far from the $1 / 3$ limit of the small deformations approximation.

The realization of the VCM uses an holosteric solution where the edge of the central active meniscus is linked with an outer rigid ring via a very thin collar having a $50 \mu \mathrm{m}$ thickness (Fig. 5), and an air pressure is applied on the back side of the meniscus. This simplifies the mounting and avoids air leak by having the mirror and its outer ring in the same sample.

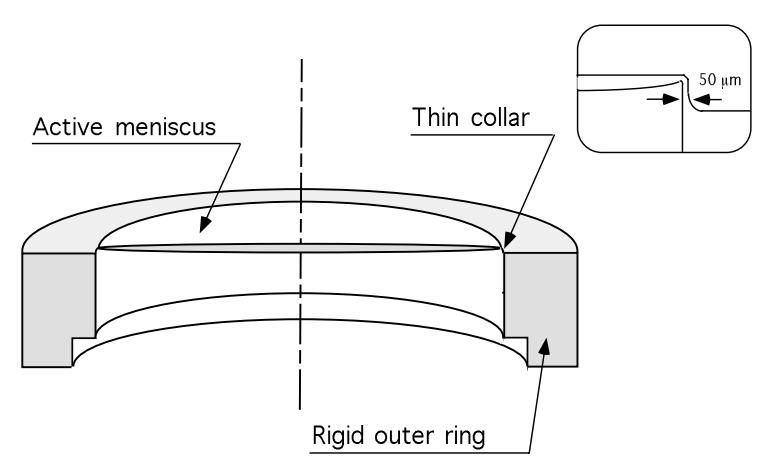

Fig. 5. Holosteric concept developed for the VCM

\section{Optical performances}

The ESO requirements asked for the VCM during operation are tight. The main one is to limit the surface deviation from a sphere to less than one wave peak-to-valley (PTV) for $\lambda=632.8 \mathrm{~nm}$, but the clear aperture concerned varies during the operation. The deviation has to be minimized on two different diameters according to successive ranges of curvatures. To radii of curvature between [2800 and $230 \mathrm{~mm}$ ] and those between [230 and $84 \mathrm{~mm}$ ] correspond respectively a $14 \mathrm{~mm}$ and a $6 \mathrm{~mm}$ clear aperture.

The important result deduced from surface measurements is that the central part of the mirror fulfils the requirements: deviation from a sphere is lower than $\lambda$ PTV on a $6 \mathrm{~mm}$ diameter. The analysis shows that on a $6 \mathrm{~mm}$ 
diameter the deviation remains below $\lambda$ during the whole curvature range (LOOM 1994), as proved by the interferogramms in Fig. 6 taken at radius of curvature equal to $2800 \mathrm{~mm}$ and $84 \mathrm{~mm}$. A large curvature variation can be achieved with a reasonable good optical quality on the central part of the surface. Unfortunately, the analysis also shows that this good quality is not achieved on the full aperture. For radii from $2800 \mathrm{~m}$ to $230 \mathrm{~mm}$ the outer part of the mirror surface is out of quality requirements, due to an excessive amount of spherical aberration.

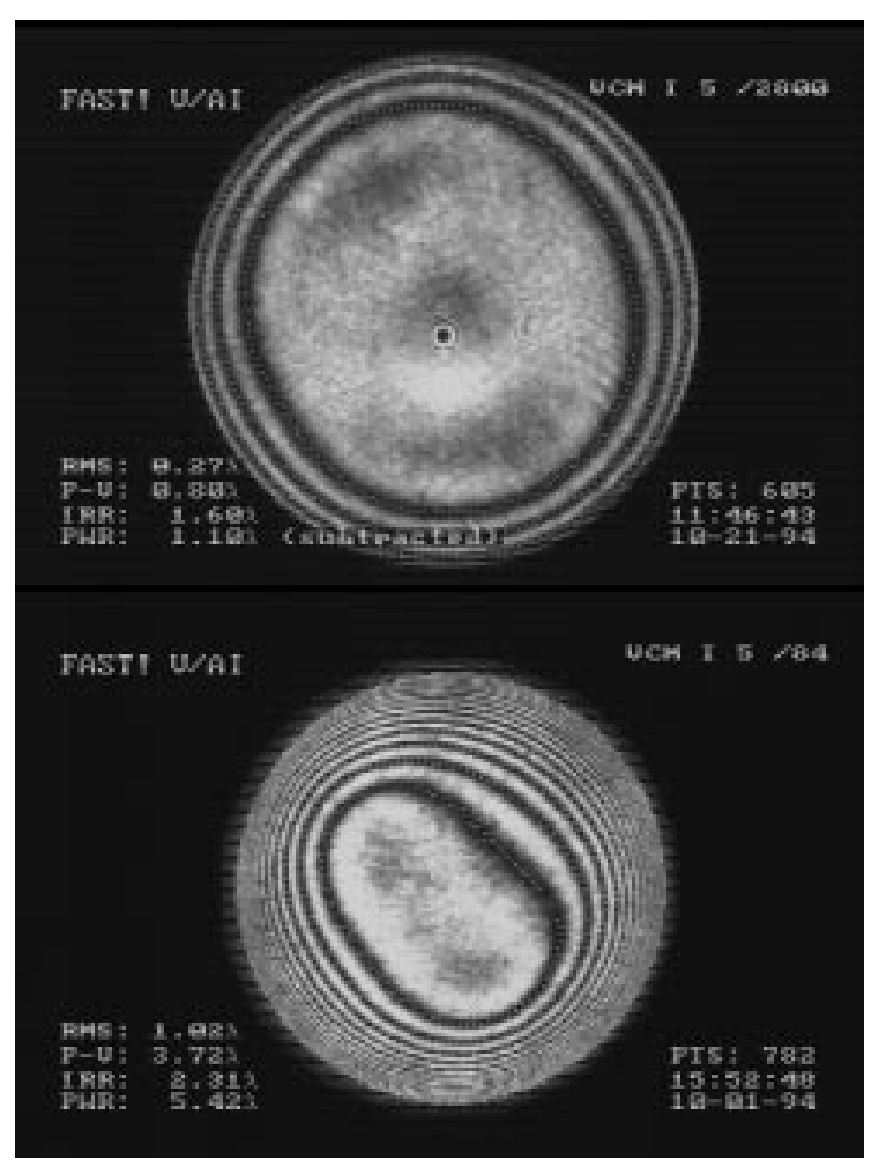

Fig. 6. Interferograms of the optical surface of a VCM sample (\#5): The radii of curvature are $2800 \mathrm{~mm}$ (up) and $84 \mathrm{~mm}$ (down). The diameters on which the deviation from a sphere is lower than $\lambda$ PTV are $15 \mathrm{~mm}$ and $7 \mathrm{~mm}$ respectively

This problem comes from two different parts:

- 1 - The collar at the edge of the active meniscus produces a "semi built-in" effect, equivalent to a rigidity increase, depending to the ratio between the collar and the meniscus thicknesses.

The bending moments distributed along the outer edge of the meniscus modify the deflection shape which is then different to the calculated spherical one.

- 2 - The linearisation of the strains in the mirror substrate during the pre-stressing stage, also called "EwingMuir" effect, changed the curvature of the mirror. This added convexity (about $15 \mu \mathrm{m}$ sag) produces a deviation from the theoretical deflection just at the outer part of the meniscus where the residual spherical aberration is observed.

A second set of mirrors was realized with an added "lens profile" which provides a $15 \mu \mathrm{m}$ overthickness at the center, and a $15 \mu \mathrm{m}$ under-thickness at the edge, that compensate for the rigidity increase introduced by the collar. The results obtained with this modified profile show that the spherical aberration is reduced by more than a factor of two on the tested samples (LOOM \& Von der Lühe 1996).

Table 1 presents the optical quality (rms and PTV)

Table 1. Wave Front Error of a VCM surface (sample \#54) on the whole curvature range

\begin{tabular}{crcrcc}
\hline \hline$P$ & $R$ & $a$ & \multicolumn{3}{c}{ Wave Fronf Error $(\lambda)$} \\
\cline { 4 - 6 }$\left[\begin{array}{c}P \\
{[\mathrm{bar}]}\end{array}\right.$ & {$[\mathrm{mm}]$} & {$[\mathrm{mm}]$} & Sph. Abb & rms & PTV \\
\hline 0.233 & 2800 & 7 & 0.492 & 0.439 & 2.271 \\
1.013 & 420 & 7 & 8.098 & 0.929 & 4.713 \\
1.815 & 230 & 7 & 16.170 & 1.481 & 7.118 \\
1.798 & 230 & 3 & 0.270 & 0.111 & 0.588 \\
3.341 & 140 & 3 & 0.219 & 0.130 & 0.679 \\
5.082 & 105 & 3 & 0.027 & 0.146 & 0.755 \\
7.561 & 84 & 3 & 0.460 & 0.202 & 0.849 \\
\hline
\end{tabular}

achieved with one of the VCM samples on the whole curvature range, the spherical aberration amount is also indicated.

For radii of curvature from $2800 \mathrm{~mm}$ to $230 \mathrm{~mm}$ the Wave Front Error (WFE) is evaluated on a $14 \mathrm{~mm}$ diameter. The spherical aberration becomes rapidly important leading to an out of requirement quality but, as already mentioned, the best results are obtained for a $6 \mathrm{~mm}$ diameter. At the $230 \mathrm{~mm}$ radius, the spherical aberration is divided by 60 when we reduce the clear aperture from 14 to $6 \mathrm{~mm}$. This amount of spherical aberration remains low within the range $[230-84 \mathrm{~mm}]$, and the WFE (PTV) is slowly increasing from $0.59 \lambda$ for $230 \mathrm{~mm}$ to $0.85 \lambda$ at 84 $\mathrm{mm}$. This proves that the largest deviations come from the outer part of the mirror. Although the spherical aberration amount has been divided by two this is still the major problem encountered.

\section{Curvature control}

The other important point related to the achievement of a coherent field at the combined focus, is the adjustement of a precise curvature during the operation of the VCMs. To evaluate this accuracy, we made multiple scans on the whole curvature range from which we calculated the mean curvatures achieved and deviations to them. This calibration is crucial for the monitoring of the VCM curvature 


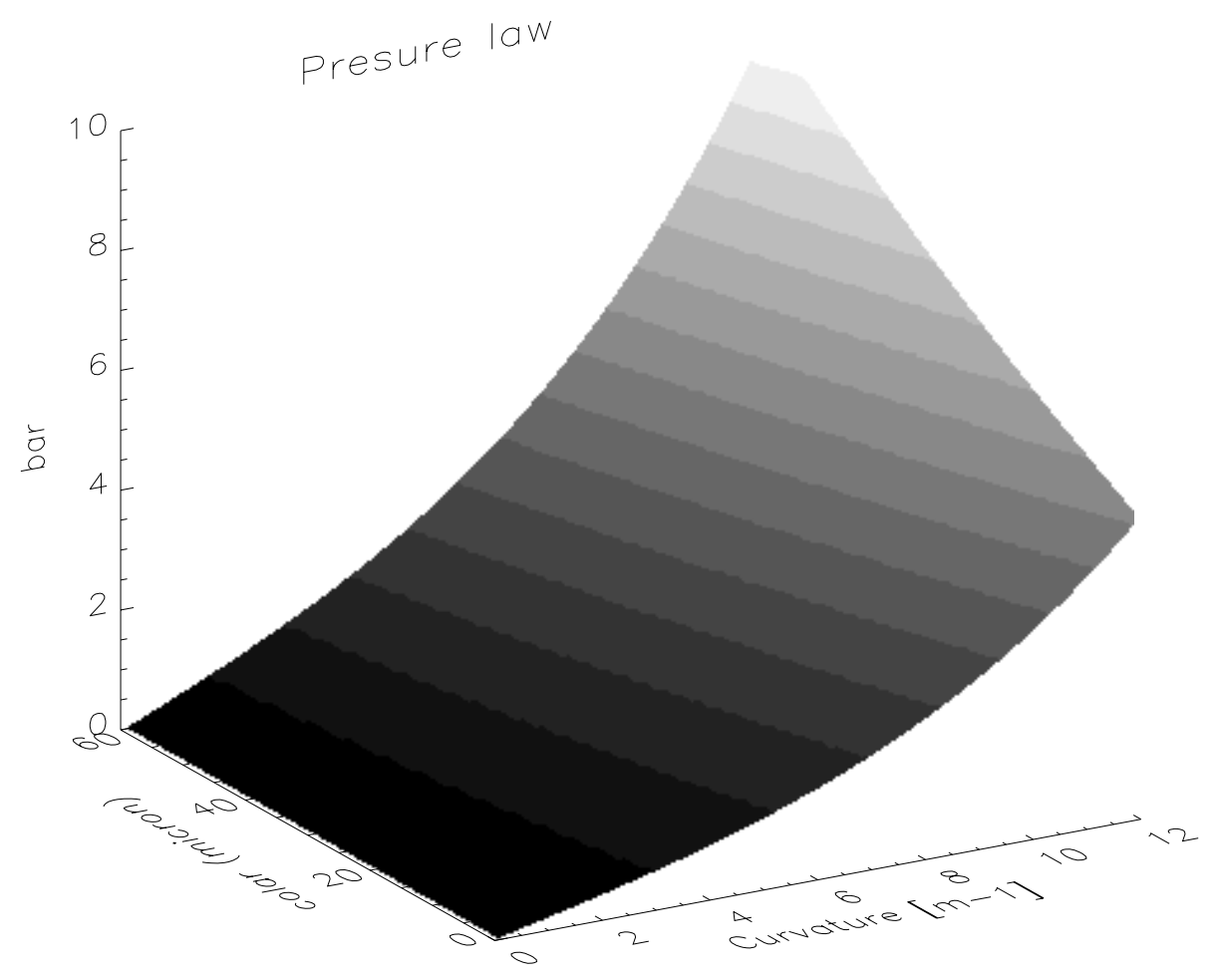

Fig. 7. Semi-clamped edge pressure law calculated for the mirror \#5

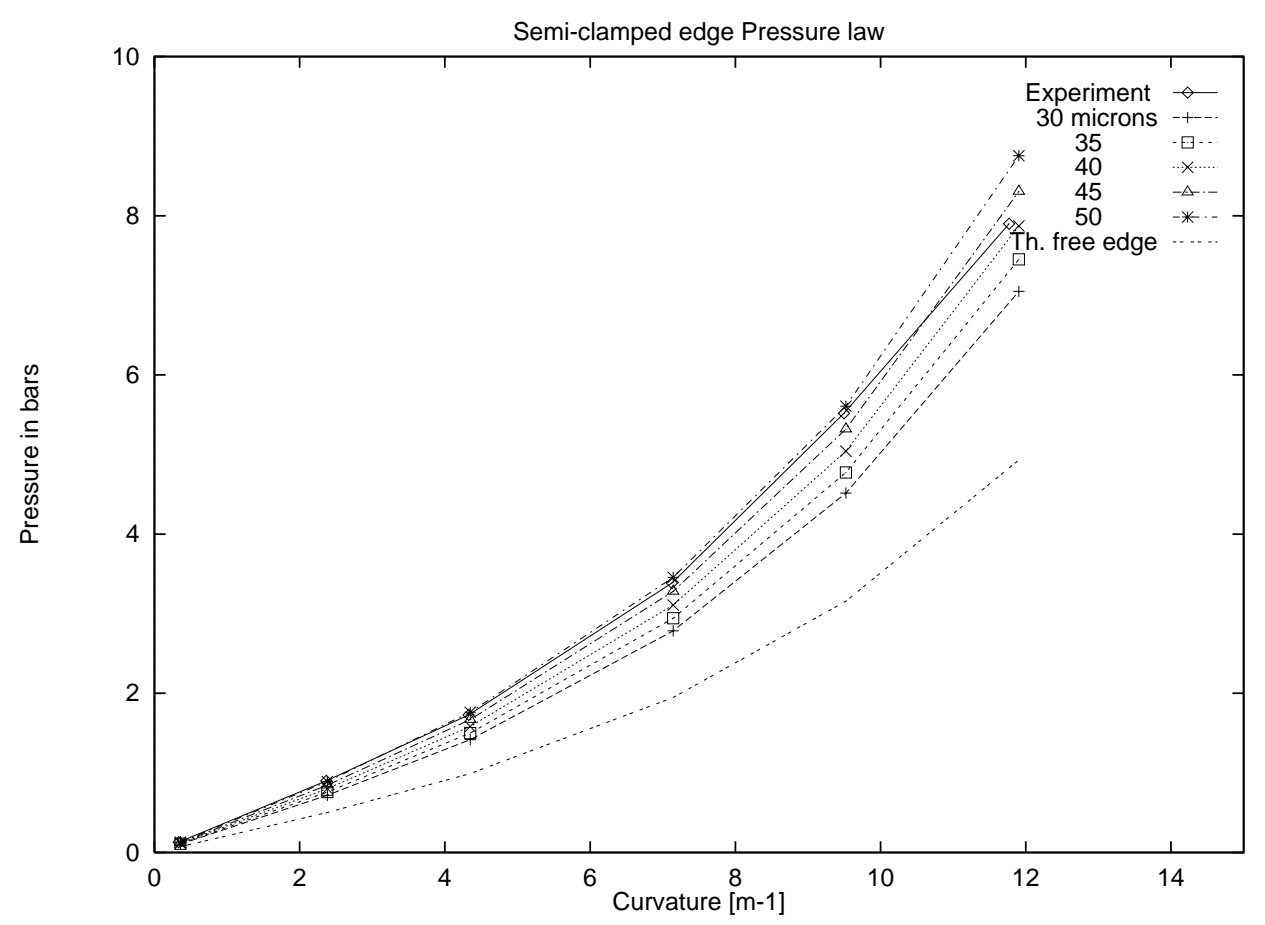

Fig. 8. Experimental pressure law compared with different collar thickness theoretical cases 
under an open-loop control. The resulting error on the curvatures is less than $510^{-3} \mathrm{~m}^{-1}$ which is the limit value accepted by ESO in the control curvature accuracy requirement. This result proves that the Pressure/Curvature relation is a tight one and permits to achieve a precise curvature with a high accuracy. The set of mean curvatures is used to modelize a Pressure/Curvature relation, using a Lagrange interpolation, which is included in the pressure monitoring software. As the relation depends of the active meniscus and the collar thicknesses, this calibration has to be done for each mirror because of small variation among the samples.

In the same time, we included the "semi-clamped edge" effect in the relation Load/Curvature displayed in Fig. 4. Where, in the free-edge case, a pressure $P_{0}$ is needed to achieve a given curvature, then a pressure $P_{\mathrm{c}}=P_{0} \cdot(1+c / t)^{3}$ is necessary for a meniscus having a central thickness $t$ and a collar thickness $c$ in the semiclamped case. The Fig. 7 presents this new relation for various collar thicknesses from a 0 (free-edge) to $60 \mu \mathrm{m}$, compared with the experiment, for one of the samples (\#5) having a central thickness of $237 \mu \mathrm{m}$ and a $50 \mu \mathrm{m}$ collar thickness. The good agreement with the theoritical predictions (Fig. 8), also verified with other samples, proves that the effect added by the collar rigidity has been properly analysed and included in the numerical simulation which is now accurate.

\section{Conclusion}

This is the first time that a variable curvature mirror is achieved with such a large range. Using large deformations theory, the maximal flexion achieved from the initial plane figure, is close to $1200 \mathrm{He}-\mathrm{Ne}$ fringes.

The achieved optical quality is in accordance with the ESO requirements for a 3.5 arcsec FOV (WFE $<\lambda$ on a $6 \mathrm{~mm}$ diam.) and leads to the realization of a VCM system fulfilling its goals in the forthcoming optical interferometric array of the VLT. The manufacturing process now represents the limit of the technology. If a wider FOV of $8^{\prime \prime}$ is planned, we will have to consider a larger active mirror in order to achieve the required optical quality on a $14 \mathrm{~mm}$ diameter, this can be obtained by using a 28 or $30 \mathrm{~mm}$ mirror. Today this VCM system is being included in the delay-line system in order to setup and calibrate the interfaces with the other devices of the interferometric mode. A gold coating, easy to apply on a stainless steel substrate, has been done on the first sample delivered to ESO in order to increase the reflectivity of the mirror.
The integration will be done during this year, and the installation on the Paranal observatory of the first complete system (delay-line with VCM) will occur around year 2000. The VLTI will not be the only interferometer to benefit of this type of device, as recently such a VCM system has been choosen to be used in another optical interferometric array. Variable curvature mirrors will be part of the upgraded Grand Interferometer à 2 Telescopes (GI2T) of the Observatoire de la Côte d'Azur, located in Plateau de Calern.

\section{References}

Beckers J.M., 1991, "Interferometric Imaging with the Very Large Telescope", J. Opt. (Paris) 22, p. 73

Connes P., 1956, Rev. Opt. 35, 37

Connes P., Michel G., 1975, Appl. Opt. 14, 9, 2067

ESO Document, 1992, "Technical Specification for a Variable Curvature Mirror.", VLT-SPE-ESO-16120-0248

Ferrari M., Lemaître G., 1993, "Analysis of large deflection zoom mirrors for the ESO VLTI", A\&A 274, 13

Ferrari M., et al., 1994, "Highly variable curvature mirrors for the VLT Interferometer", Proc. SPIE Conference on Astronomical Telescopes and Instrumentation for the 21st Century, 2201, Kona - Hawaii, p. 811

Ferrari M., et al., 1995, "Realization of a highly variable curvature mirrors for the VLT Interferometer: Testing and Results", Proc. SPIE Conference on Optical Testing and Hologr. 2576, Tokyo, p. 270

Jörck H., et al., 1992, "The Design of the Delay Lines of the VLT Interferometer.", Proc. ESO Conference on High Resolution Imaging by Interferometry, Beckers J.M. and Merkle F. (eds.)

Lemaître G., 1976, "Elasticité et miroirs à focale variable", Comptes rendus Acad. Sc. Paris, 282B, p. 87

LOOM, 1991, "Phase A study report on zoom mirrors", ESO doc.

LOOM, 1994, "Phase B study report on zoom mirrors", ESO doc.

LOOM \& Von der Lühe O., 1996, "Phase C study report on zoom mirrors", ESO doc. VLT-TRE-ESO-15220-1055

Timoshenko S., Woinowski-Krieger S., 1959, "Theory of Plates and Shells". McGraw-Hill (ed.)

Von der Lühe O., et al., 1992a, "The configuration of the VLT Interferometer on the Paranal Site", Proc. ESO Conference on High Resolution Imaging by Interferometry, Beckers J.M. and Merkle F. (eds.)

Von der Lühe O., et al., 1992b, "Interferometry with the Very Large Telescope", Proc. ESA Workshop on Solar Physics and Astrophysics at Interferometric Resolution, Paris, p. 247 